\title{
Skin Pharmacology and Physiology
}

Skin Pharmacol Physiol , DOI: 10.1159/000517906

Received: September 14, 2020

Accepted: June 8, 2021

Published online: June 21, 2021

\section{Lower limb dynamic activity significantly reduces foot skin perfusion- exploring data with different optical sensors in age-grouped healthy adults} Florindo M, Nuno SL, Rodrigues LM

ISSN: 1660-5527 (Print), elSSN: 1660-5535 (Online)

https://www.karger.com/SPP

Skin Pharmacology and Physiology

\section{Disclaimer:}

Accepted, unedited article not yet assigned to an issue. The statements, opinions and data contained in this publication are solely those of the individual authors and contributors and not of the publisher and the editor(s). The publisher and the editor(s) disclaim responsibility for any injury to persons or property resulting from any ideas, methods, instructions or products referred to the content.

Copyright:

(C) S. Karger AG, Basel 
Lower limb dynamic activity significantly reduces foot skin perfusion-exploring data with different optical sensors in age-grouped healthy adults Margarida Florindo ${ }^{1,2}$, Sérgio Loureiro Nuno ${ }^{1,3,4}$, and Luis Monteiro Rodrigues ${ }^{1 *}$

${ }^{1}$ Universidade Lusófona CBIOS - Research Center for Biosciences and Health Technologies, Av Campo Grande, 376, 1749- 024, Lisboa, Portugal

${ }^{2}$ ESSCVP the Portuguese Red Cross Health School. Dep. Physiotherapy | Lisboa, Portugal

${ }^{3}$ Clínica S João de Deus - CTD | Lisboa Portugal

${ }^{4}$ Escola Superior de Tecnologia da Saúde de Lisboa -ESTeSL Lisboas' Polytechnic Institute | Lisboa, Portugal

Short title: Foot microcirculation changes following gait

* corresponding author

Luis Monteiro Rodrigues

Universidade Lusófona CBIOS - Research Center for Biosciences and Health Technologies, Av Campo Grande, 376, 1749- 024, Lisboa, Portugal

monteiro.rodrigues@ulusofona.pt

number of tables: $\quad 2$

number of figures $\quad 3$

word count:

KEYWORDS: walking gait; dorsal foot microcirculation; laser Doppler flowmetry; photopletismography; polarised spectroscopy

\section{ABSTRACT}

Introduction. The human lower limb is widely used as a model to study in vivo microcirculatory physiology and pathophysiology. It is a preferential target for critical co-morbidities (overweight, diabetes, peripheral vascular disease). Movement and activity are consistently regarded as beneficial, but the related adaptive physiology is still poorly understood. Our goal was to better identify the foot microcirculatory changes after a regular walking gait activity in healthy subjects of different ages. Methods. Twelve healthy participants of both sexes, with normal Body Mass Index (BMI )and Ankle-Brachial Index, were selected and grouped according to age - Group I (21.0 \pm 1 y.o.) and Group II (55.8 \pm 3 y.o.). The protocol involved two phases of 5 minutes duration each - Phase 1, a static standing position, and Phase 2, 5 min walking with a comfortable pace on a pre-established circuit. Perfusion changes were assessed in the dorsal region of both feet before (baseline, Phase 1) and after the gait period (Phase 2) by non-invasive optical technologies - Laser Doppler flowmetry (LDF), photoplethysmography (PPG) and polarised spectroscopy (PSp). Comparative statistics were performed with a $95 \%$ confidence level. Results. All instruments detected an asymmetric non-significant perfusion between right and left feet during rest in all participants with values in females consistently lower than men. Older participants exhibited lower baseline values than the younger group. Gait evoked a perfusion reduction in all participants relative to Phase 1 detected with all technologies, with statistically significant changes recorded with LDF (Group I $p=0.033$ and Group II $p=0.028$ ) and PSp (Group II $p=0.041$ ). Furthermore, LDF revealed that gait significantly reduced perfusion velocity in the older group $(p=0.003)$. Corresponding changes in the younger group were present but discrete. Recovery to baseline levels was also slower in the older group. Discussion/Conclusions. Our results confirm that perfusion is age dependent and demonstrates the clinical relevance of simple dynamic activities such as gait. This reduction of the dorsal foot perfusion occurs in depth, being more pronounced with the movement intensity, suggesting a wide application potential in early diagnostics as for rehabilitation. 
Introduction

The standard bipedal nature of human movement determines a complex adaptive environment between muscular activity and haemodynamics, even for daily life routines, that is still poorly understood. Human models have been central to look into many of these relationships (1-4) - the use of in vitro models is not that common, although some recent functional models have been developed to assess the impact of perfusion on physiological mechanisms (5). We understand the perfusion reduction in the leg when passing from lying down to sitting (1), from which the edema mechanism of the foot and ankle, a normal complication of short duration weight displacements, was depicted (2-4). Other adaptive perfusion features related to posture $(6,7)$ or different dynamic activities have also been progressively described (8-12). Conversely, the progressive reduction of activity associated with ageing (also related to sarcopenia) and microcirculatory impairment associated with peripheral vascular disease (PVD) inflammation or diabetic microangiopathy always accelerate frailty $(13,14)$.

Research on these thematics have been mostly driven within the perspective of exercise and rehabilitation in very specific contexts. Nevertheless, mechanisms responsible for the benefits relating exercise and circulation, especially in the lower limb, are far from being clearly characterized (9-12). An insufficient number of randomized clinical trials and the use of a broad array of procedures (type of exercise/activity, duration, frequency), combined with different technological options and a diversified interpretation of variables have challenged consensus on involved mechanisms. In the diabetic patient, exercise seems to have positive effects on lower limb hemodynamics (15-17) but in the presence of peripheral arterial disease, results seem to depend on the type of exercise and the severity of the existing lesions (17-19). In PVD patients, the impact of exercise seems to be even more difficult to establish, except in more moderate cases when claudication is stabilized $(18,19)$. It is interesting to note that even in the absence of a specific pathology, the adaptation of the microcirculatory physiology of the lower limb and foot according to movement/exercise is not clearly established $(8,11,20)$. However, the decrease in walking speed is recognized as a useful indicator of impairment related to the multi-morbidity associated with ageing $(20,21)$.

The negative impacts of the lack of movement/motor activity on our normal physiology are known, either with sedentarism or related to some types of cerebrovascular accident or brain trauma (20-23). Passive leg movement alone has been indicated to stabilize circulation in the lower limb and prevent syncope in healthy adults $(21,22)$. Therefore, while we accept that movement and exercise have true clinical relevance which contributes to the preservation of health and well-being, we further believe there is a close relationship between movementposture and peripheral perfusion which adapts differently in different dynamic conditions. These aspects of body dynamics physiology and its conditioning factors, including age, must be better known.

Under this view, the present study aims to explore a) the perfusion changes resulting from a normal short duration gait activity in b) two groups of healthy individuals of different ages using various light sensors measuring perfusion at different skin depths. Finally, we also aim c) to propose a reproducible experimental design planned to evaluate and compare these relationships as close as possible to the normal physiological state in order to look deeper into these aspects of integrative physiology.

\section{METHODS}

This exploratory study involved a convenience sample of twelve healthy participants selected according to predefined inclusion / non-inclusion criteria, primarily comprised of students and collaborators within our research unit. All participants reported regular physical activity and absence of any metabolic disease (diabetes, dyslipidemia). Vascular status was evaluated by the ankle-arm index ( $\mathrm{ABI}$ ), a common indicator of vascular health $(23,24)$, and Body Mass Index (BMI) values were within the normal range. Participants were normotensive, nonsmokers, and free of any medication or food supplementation. Caffeine and alcohol consumption was prohibited 24 hours prior to measurements, as well as the use any topical (including cosmetic) application in the assessment areas. All young women reported regular menstrual cycles. Female participants were not using oral or implanted contraceptives.

Participants were divided by age. Group I included six young adults (three women and three men, $21.0 \pm 1$ years old and BMI of $22.7 \pm 1.6 \mathrm{~kg} / \mathrm{m}^{2}$, and Group II six older adults (three women and three men) with $55.8 \pm 3$ years old and BMI of $24.8 \pm 1.9 \mathrm{~kg} / \mathrm{m}^{2}$, values adjusted for age (25).

All participants were duly informed about the objectives of the study, including all methods and technologies to be used. Procedures followed the principles established by the Helsinki Declaration and subsequent amendments (26) and were assessed in advance by the Institutional Ethics Committee. 
Measurements were done under controlled conditions, including room temperature $\left(25 \pm 1^{\circ} \mathrm{C}\right)$, humidity ( $40 \%$ $60 \%$ ), and uniform lighting, to minimize any environmental effects on participants and measuring systems. After 10 minutes of adaptation in the standing position, the protocol was sequenced in two phases. In Phase 1, baseline recordings were obtained over 5 minutes with participants static while standing with feet separated $10 \mathrm{~cm}$ apart. A period of walking activity followed in a pre-established circuit in the laboratory area for 5 minutes at a comfortable pace. The participants then resumed the starting position, standing still for 5 minutes to register post-gait recovery (Phase 2). Probes were removed for the walking period and quickly replaced in the same sites once the activity ended.

Perfusion-related variables were obtained from the dorsal region of both feet, an option demonstrated to reduce signal variability $(27,28)$. Three optical technologies were used:

- Laser Doppler flowmetry (LDF) [Perimed PF5010 System, Stockholm, Sweden], in which the "Doppler effect" obtained with red light enables local perfusion to be calculated $(29,30)$ from the speed and number of particles in movement (mainly red blood cells) reflecting this light. Data is quantified in terms of blood perfusion (BP expressed in arbitrary units BPU's), concentration of red blood cells (CRBC also expressed in arbitrary units AU's) and velocity (in $\mathrm{cm} / \mathrm{sec}$ ). The system also allows continuous monitoring of skin temperature, known to affect circulation, particularly in the extremities.

- Photoplethysmography (PPG) [BITalino kit, PLUX Biosignals, Lisbon, Portugal], which allows the measurement of small variations of the blood perfusion volume (BPV) (28-31) through the reflected green light, expressed in arbitrary units. The PPG wave is a synchronous heartbeat-related pulse wave (AC) and represents the volume of blood generating the wave $(30,31)$.

- Polarized light spectroscopy (PSp), using the Tissue Viability Imaging ${ }^{\circledR}$ (TiVi) system [TiVi8000, WheelsBridge, Sweden], which permits the calculation of the concentration of erythrocytes (CRBC) in arbitrary units (AU) as evaluated throughout a sequence of photographs taken in the same region of interest (ROI) $(32,33)$.

These optical technologies use light with different wavelengths, providing different interactions with the tissue (34) (Figure 1), which must be considered in the analysis and interpretation of results.

Heart rate (PR) and blood pressure (BP) were also monitored with a digital sphygmomanometer [Pic

22012000200 Esfigm Classic Check, Artsana S.p.A, Italy].

In Phase 1, the perfusion-related variables were obtained in the minute immediately before beginning the activity, regarded as the baseline reference. For the recovery period analysis (Phase 2), measurements took place after replacing all measuring probes, approximately one minute after the gait period. As mentioned, all probes were removed during the gait period and replaced in the recovery.

In order to denoise and improve the quality of the PPG recordings, a MatLab (Mathworks Inc., Natick, MA, USA) filter was applied. This software selects the dominant wave acquired to calculate a probable distribution of the pulses of interest, improving accuracy without changing the validity of the signal (35).

Data were analyzed through descriptive and comparative statistics using SPSS v.22.0 software (IBM Corp. Amrock, NY, USA). Data normal distribution was previously tested in both groups by the Shapiro Wilk's test (Figure 2). The intraindividual perfusion variations (right and left foot) as the interindividual comparisons were evaluated using the Levene's test for variance equality and with the " $t$ test" for comparing means. The Wilcoxon sign-rank test was used for comparison between phases in each group. A 95\% confidence level was adopted.

\section{RESULTS AND DISCUSSION}

Table 1 summarizes the global data set. Results are shown as mean \pm standard deviation.

The microcirculatory adaptation in orthostatic or semi-static variations have been described. A prolonged standing position evokes the mobilization of considerable amounts of blood to the more distal regions of the lower limbs. This mobilization decreases the venous return and activates pressure receptors as part of a wide and complex interaction between different (central and peripheral) physiological sensors and effectors still far from being fully identified and understood $(4,6,12,28)$. The perfusion adaptation related to movement such as walking or running is less known and depicted $(3,10,21)$. Most of the studies use laser Doppler flowmetry (LDF) as a reference method $(7,29)$. Only a few have chosen photoplethysmography $(P P G)(7,30)$ as the measurement instrument, and, to the best of our knowledge, only our group hasapplied polarised spectroscopy to further explore microcirculatory physiology and skin flowmotion $(32,33)$. In most studies, one single instrument is used to measure perfusion. Although based on similar optical principles, each technology uses different wavelength/light sources, which results in different interactions between light and tissue, i.e., different microcirculatory information $(34,36,37)$, making comparisons of results impossible. By operating with these three systems, we 
expect to fully characterize all in vivo changes occurring as a consequence of gait in both groups and identify differences, if any, attributable to age. A recently published study (37) suggested that light penetration into the skin is a direct function of the wavelength used. The skin penetration capability of LDF is thought to be deeper than that of PPG, close to $1 \mathrm{~mm}$ (37). In contrast, polarized light spectroscopy results from the interaction of white, incoherent, filtered light with the skin surface (31). It detects red blood cell concentration and not perfusion itself, operating in the most superficial layers of the skin.

At rest (Phase 1), all technologies detected asymmetries between the perfusion-related variables from the left and right feet of each participant. This bilateral perfusion asymmetry was expected and reported as nonsignificant in healthy subjects (38) as in our groups. A recent study suggested this variation results from small morphometric variations of the femoral artery with haemodynamical impact in paired legs (39). As shown in Table 1 , these differences disappear with movement, likely as a result of the adaptive cross-talk between movementposture and hemodynamic sensors and effectors. These intraindividual variations also found in the mouse (27) have been called "physiological" to distinguish from those that may originate from asymmetric vascular pathology of the lower limbs.

In line with these observations, all systems detected a perfusion reduction in the post-gait period (Phase 2) following movement in both groups. In (the younger) Group I, a significant reduction difference $(p=0.033)$ could be detected in this period with LDF perfusion (Table 1). In Group II, significant perfusion reduction differences in the post-gait period could be detected by LDF perfusion $(p=0.028)$ and velocity $(p=0.003)$, as by PSp concentration of red blood cells $(p=0.041)$. Despite the PPG "denoising" procedure, this instrument could not detect significant changes in any of the groups. All systems confirmed that by the end of the experiments, perfusion had not returned to normal reference values in any of the groups (Table 1). A non-significant increase in systolic and diastolic blood pressure was also detected in Phase 2 in both groups.

No local temperature variations were detected under the studied conditions.

This original aspect of our setting, detecting the same outcome with three sets of optical sensors - LDF PPG and PSp - clearly suggests that these significant perfusion reductions can only be a consequence of gait. The decrease of the more superficial blood flow, revealed by CRBC (PSp) as well as the reduction of the deeper flow means an effective mobilization of blood from surface to the deeper effector motor units. The resulting blood sequestration at the thigh and leg effector muscles provides an increased metabolic activity of those motor units $(10,40)$.

Our group recently introduced the study of microcirculatory perfusion dynamics in the human lower limb with the "step in place" model (33), applying a similar experimental design with PPG and PSp as measurement technologies. In these studies, PPG only allowed the measurement before and after the movement and detected no differences of statistical significance. As in the present study, however, the PSp system revealed a significant decrease $(p<0.007)$ of the TiVi index (CRBC) following movement, which remained significantly below the base value $(p<0.028)$ in the recovery phase. Pulse rate $(p<0.005)$ and blood pressure increased, with significance only for systolic pressure $(p<0.003)$. This study also suggested that a semi-dynamic task such as the step-in-place might effectively evoke a lasting response, perhaps not far from that observed with dynamic movements such as gait.

To explore those age-related differences previously referenced, we further compared the most representative perfusion-related variables obtained in both groups by LDF and PSp. Each participant limb pair was transformed into a single normalized value corresponding to the mean $( \pm s d)$ to reduce variability and facilitate analysis (Table 2). As shown, changes in both phases do not substantially differ between age groups, and gait promotes an equivalent statistically significant reduction of perfusion (LDF) in both groups $(p=0.046)$. However, significant reductions of blood velocity ( $p=0.028)$ detected by LDF, and of CRBC $(p=0.046)$ detected by PSp following gait became very clear. It is known that even in the absence of disease, ageing involves a progressive stiffening of all circulatory structures from macrocirculation (arteries) to microcirculation. This increases the pulsatile stress at microcirculation and reduces flow velocity (41). Furthermore, we have noticed that the younger group shows higher predominance of one foot perfusion over the other (right-left perfusion ratio analysis, data not shown). Thus, we cannot exclude the (non-significant) perfusion asymmetry detected between limbs in Phase I as a contributor. However, more information (e.g., load dominance, fine movement preference, vascular anatomy) is needed to better examine this interesting direction.

Our results have also shown that values in female subjects were consistently lower than in males, except for CRBC, even if not statistically significant. Gait evoked the described reduction of these variables, again with no statistical expression. Figure 3 shows the perfusion-related changes registered in both sexes using, as before, the 
mean ( $\pm s d)$ of the most representative variables to facilitate our analysis. Differences in prevalence, expression, and prognosis in cardiovascular diseases between men and women are known (42), although gender differences in vascular and cardiac physiology have been difficult to demonstrate in healthy individuals. Perfusion-related differences between sexes have been described in exercise training and resistance, likely reflecting some differences in skeletal muscle perfusion and performance $(43,44)$. However, some sex-related differences seem to exist determining different performance fatigabilities. This seems to be particularly relevant in clinical terms since it underlines the need to ensure adequate "dosages" in exercise as in rehabilitation to promote adequate integrative responses that might not be present (in women) if that dosage is suboptimal $(43,45)$.

Our results are in line with previous publications demonstrating the importance of this movement-induced blood mobilization which seems to be proportional to the intensity of the movement, i.e., more obvious with gait than with the "step in place" activity $(21,33)$. The clinical utility of this mobilization could be considerable, as it demonstrates that even a low-intensity activity, far from a rigorous training program, broadly impacts microcirculatory function. This may offer a more feasible pathway to help prevent the multiple impacts of ageing, including sarcopenia, or to reduce microcirculatory stress, e.g., of diabetes. We believe these findings are also potentially relevant for early diagnosis of gait-related abnormalities underlining the importance of personalized rehabilitation programs.

\section{CONCLUSIONS}

By identifying this significant foot skin perfusion reduction resulting from regular gait, we demonstrate the clinical importance of dynamic movement as a vascular preventer / promoter of particular importance under the current trends of personalized care, including home health. Our findings also draw attention to the interindividual perfusion asymmetries between limbs at rest, more pronounced in the younger group participants, indicating potential new research directions related to posture and movement preferences. Age is a major determinant modifying the response adaption and reducing asymmetries even in the absence of pathology. Analysis also suggests different requirements for men and women, another aspect to consider in personalized care. Our methodological proposal is technically sound and reproducible, applicable to different conditions such as movement time-duration/load exposure. We also estimate that this design, kept as close as possible to the normal physiological state, will be easily adapted to other patient profiles (e.g., overweight), and applicable to different conditions involving microcirculatory impairment. Results comparisons are crucial to look deeper into these adaptive mechanisms, and the methodology used in this exploratory study meets that requirement, suggesting a viable analytical approach to microcirculatory treatment and research.

\section{Acknowledgement}

The authors are grateful to the participants, to our colleague João Gregorio and to all who contributed to the technical analysis, editing, and proof reading of the manuscript .

\section{Statement of Ethics}

As previously stated, all procedures followed the principles established by the Helsinki Declaration and subsequent amendments for all phases of the experimental design. An informed written consent was made available and explained to all participants prior to inclusion. The protocol was previously screened by the university's Health School Ethic Committee (authorization CEECTS.05.2013).

\section{CONFLICT of interest}

The authors have no conflicts of interest to declare.

\section{FUNDING}

This study was funded by FCT - Fundação para a Ciência e Tecnologia IP, through grants UIDB/04567/2020 and UIDP/04567/2020

\section{Author's Contributions}

MF, methodology, investigation, data curation, draft preparation; SN investigation, data

curation; LMR Conceptualization, methodology, writing, reviewing and supervision. 


\section{REFERENCES}

1. Amery, A., Bossaert, H., Deruyttere, M., Vanderlinden, L,. \& Verstraete, M. Influence of Body Posture on Leg Blood Flow. Angiologica; 1973; 10:152-163 doi.org/10.1159/000157968

2. Winkel, J., \& Jørgensen K. Evaluation of foot swelling and lower-limb temperatures in relation to leg activity during long-term seated office work, Ergonomics, 1985; 29:2, 313-328. DOI: 10.1080/00140138608968267

3. Cloughley W.B., \& Mawdsley R.H. Effect of Running on Volume of the Foot and Ankle JOSPT. 1995; 22, 4, 151-154. DOI: 10.2519/jospt.1995.22.4.151

4. Guyton, A.C., \& Hall, J.E.,Diuretics and Kidney Diseases in "Textbook of medical physiology", WB Saunders, Philadelphia, chapter 31, p. 404-413. 2000

5. Strüver K, Friess W, Hedtrich S: Development of a Perfusion Platform for Dynamic Cultivation of in vitro Skin Models. Skin Pharmacol Physiol 2017;30:180-189. doi: 10.1159/000476071

6. Truijen, J., Kim, Y.S., Krediet, C.T.P., Stok, W.J., Kölgen, R.S., Colier, W.N., et al. Orthostatic leg blood volume changes assessed by near-infrared spectroscopy. Experimental Physiology, 2012; 97: 353 361. https://doi.org/10.1113/expphysiol.2011.061051

7. Silva, H., Ferreira, H., Bujan, M.J., \& Rodrigues, LM., Regarding the quantification of peripheral microcirculation - Comparing responses evoked in the in vivo human lower limb by postural changes, suprasystolic occlusion and oxygen breathing. Microvascular Research. 2015; 99, 110-117. DOI: 10.1016/j.mvr.2015.04.001

8. Green, D.J., Spence, A. Halliwill, J.R., Cable, N.T., \& Thijssen, D.H. Exercise and vascular adaptation in asymptomatic humans. Exp Physiol. 2009; 96.2 pp 57-70. DOI: 10.1113/exphysiol.2009.048694

9. Padilla, J., Simmons, G.H., Bender, S.B., Arce-Esquivel, A.A., Whyte, J.J., \& Laughlin, M.H. Vascular Effects of Exercise: Endothelial Adaptations Beyond Active Muscle Beds. Physiology (Bethesda). 2011; 26(3): 132145. doi:10.1152/physiol.00052.2010.

10. Joyner, M. J., \& Casey, D. P. Regulation of increased blood flow (hyperemia) to muscles during exercise: a hierarchy of competing physiological needs. Physiological reviews. 2015; 95(2), 549-601. doi.org/10.1152/physrev.00035.2013

11. Constantini, K., Stickford, A.S., Bleich, J.L., Mannheimer, P.D., Levine, B.D., \& Chapman R.F. Synchronizing Gait with Cardiac Cycle Phase Alters Heart Rate Response during Running. Med Sci Sports Exerc , 2018; 50(5): 1046-1053. DOI: 10.1249/MSS.0000000000001515

12. McGarr G, W, Fujii N, Muia C, M, Nishiyasu T, Kenny G, P: Intradermal Administration of Atrial Natriuretic Peptide Attenuates Cutaneous Vasodilation but Not Sweating in Young Men during Exercise in the Heat. Skin Pharmacol Physiol 2020;33:86-93. doi: 10.1159/000505300

13. Stout MB, Justice JN, Nicklas BJ, and Kirkland JL., Physiological Aging: Links Among Adipose Tissue Dysfunction, Diabetes, and Frailty. Physiology 2017; 32:1, 9-19. doi.org/10.1152/physiol.00012.2016

14. Hanssen SCA, Hendriks AGM, Keijsers RMC, van Erp PEJ, van der Vleuten CJM, Seyger MMB, van de Kerkhof PCM. Response of the Endothelium to the Epicutaneous Application of Leukotriene B4. Skin Pharmacol Physiol. 2017;30(6):306-314. doi: 10.1159/000481204.

15. Mayrovitz, H.N., \& Larsen, P.B. Functional microcirculatory impairment: a possible source of reduced skin oxygen tension in human diabetes mellitus. Microvasc Res. 1996; 52: 115-126.

16. Olver, T.D., \& Laughlin, M.H. Endurance, interval sprint, and resistance exercise training: impact on microvascular dysfunction in type 2 diabetes. Am J Physiol Heart Circ Physiol 2016; 310: H337-H350. doi:10.1152/ajpheart.00440.2015.

17. Williams, D.T., Harding, K.G., \& Price, P.E.The influence of exercise on foot perfusion in diabetes. Diabetic Medicine: a Journal of the British Diabetic Association. 2007; Oct;24(10):1105-1111. DOI: 10.1111/j.14645491.2007.02218.x.

18. Olin, J. W., \& Sealove, B. A. Peripheral artery disease: current insight into the disease and its diagnosis and management. Mayo Clinic Proceedings, 2010; 85(7), 678-692. doi.org/10.4065/mcp. 2010.0133

19. Rosenblum, J., Gimmelreich, D., Rosenblum, S. Sequential contraction compression therapy raises foot temperatures in patients with peripheral arterial disease. Journal of Vascular Diagnostics and Interventions. 2016; 4: 39-43

https://doi.org/10.2147/JVD.S115169

20. Gardner, A.W., Katzel, L.I., Sorkin, J.D., Bradham, D.D., Hochberg, M.C., Flinn, W.R.. et al. Exercise Rehabilitation Improves Functional Outcomes and Peripheral Circulation in Patients with Intermittent 
Claudication: A Randomized Controlled Trial. Journal of the American Geriatrics Society, 2001; 49: 755762. https://doi.org/10.1046/j.1532-5415.2001.49152.x

21. Rosso A.L., Sanders, J.L., Arnold, A.M. Boudreau, R.M., Hirsch, C.H., Carlson, M.C., et al., Multisystem Physiologic Impairments and Changes in Gait Speed of Older Adults, The Journals of Gerontology: Series A, 2015; Volume 70, Issue 3, March, Pages 319-324. doi.org/10.1093/gerona/glu176

22. Menard, J.R., Smith, H.E., Riebe, D., Braun, C.M., Blissmer, B., \& Patterson, R.B. Long-term results of peripheral arterial disease rehabilitation. Journal of Vascular Surgery. 2004; 39, 6. https://doi.org/10.1016/j.jvs.2004.01.034

23. Aboyans, V., Criqui, M.H., Abraham, P., Allison, M.A., Creager, M.A., Diehm C., et al., Measurement and interpretation of the ankle-brachial index: a scientific statement from the American Heart Association. Circulation 2012; 126: 2890-2909. doi:10.1161/CIR.0b013e318276fbcb.

24. Stivalet, O., Paisant, A., Belabbas, D., Omarjee, L., Le Faucheur.,A., Landreau, P., et al. Exercise testing criteria to diagnose lower extremity peripheral artery disease assessed by computed-tomography angiography. PLoS ONE 2019; 14 (6): e0219082. doi.org/10.1371/journal. pone.0219082

25. Khan, S.S., Ning, H., Wilkins, J.T., Allen, N., Carnethon, M., Berry, J.D., et al., Association of Body Mass Index With Lifetime Risk of Cardiovascular Disease and Compression of Morbidity. JAMA Cardiol. 2018; Apr; 3(4): 280-287. doi: 10.1001/jamacardio.2018.0022

26. World Medical Association. World Medical Association Declaration of Helsinki: ethical principles for medical research involving human subjects. JAMA 2013.310: 2191-2194, doi:10.1001/jama.2013.281053

27. Rodrigues, L.M., Silva, H., Ferreira, H, Renault, M-A. \& Gadeau, A-P. Observations on the perfusion recovery of regenerative angiogenesis in an ischemic limb model under hyperoxia. Physiol Rep 2018; 6: e13736. doi:10.14814/phy2.13736.

28. Rodrigues, L.M., Rocha, C., Ferreira, H.A. \& Silva, H.N. Lower limb massage in humans increases local perfusion and impacts systemic hemodynamics, J Appl Physiol 2020; 128: 1217-1226. doi:10.1152/japplphysiol.00437.2019

29. Tulevski, I.I., Ubbink, D.T. \& Jacobs, M.J.. Red and Green Laser Doppler Compared with Capillary Microscopy to Assess Skin Microcirculation in the Feet of Healthy Subjects Microvascular Research. 1999; 58, 83-88. Article ID mvre.1999.2159, available online at http://www.idealibrary.com. doi.org/10.1006/mvre.1999.2159

30. Bergstrand, S., Lindberg, L.G., Ek, A.C., Lindén, M. \& Lindgren, M. Blood flow measurements at different depths using photoplethysmography and laser Doppler techniques. Skin Res Technol 2009; 15: 139-147. doi:10. 1111/j.1600-0846.2008.00337.x.

31. Allen, J. Photoplethysmogrphy and it application in clinical physiological measurement. Physiological Measurement. 2007; Mar;28(3):R1-39. Epub Feb 20. doi:10.1088/0967-3334/28/3/R01

32. Florindo, M., Nuno, S. \& Rodrigues, LM., The march in place activity - a view from the local concentration of red blood cells (CRBC) in the human foot dorsum. Biomed Biopharm Res. , 2019; (16) 1: , 62-69 DOI: 10.19277/bbr.16.1.199

33. Florindo, M., Nuno, S. \& Rodrigues, LM., Studying the human lower limb perfusion dynamics with the step in place model. Biomed Biopharm Res. 2019; (16) 2: , 195-201. DOI: 10.19277/bbr.16.2.212

34. Rodrigues, L.M., Rocha, C., Ferreira, H. \& Silva, H. Different lasers reveal different skin microcirculatory flowmotion - data from the wavelet transform analysis of human hindlimb perfusion. Sci Rep 2019; 9: 16951. doi:10.1038/s41598-019-53213-2

35. Liang, Y., Elgendi, M., Chen, Z \&, Ward, R. Analysis: An optimal filter for short photoplethysmogram signals. Scientific Data 2018; 5:180076 | doi: 10.1038/sdata.2018.76

36. Ash, C., Dubec, M., Donne, K. \& Bashford, T. Effect of wavelength and beamwidth on penetration in lighttissue interaction using computational methods. Lasers Med Sci 2017; 32, 1909-1918, https://doi.org/10.1007/s10103-017-2317-4.

37. Rodrigues, L.M., Rocha, C., Ferreira, H. \& Silva, H. Different lasers reveal different skin microcirculatory flowmotion - data from the wavelet transform analysis of human hindlimb perfusion. Sci Rep 2019; 9: 16951. doi:10.1038/s41598-019-53213-2

38. Recek, C. Calf pump activity influencing venous hemodynamics in the lower extremity. Int J Angiol. 2013 Mar;22(1):23-30. doi: 10.1055/s-0033-1334092 
39. Marcinkevics Z, Lukstina Z, Rubins U, Grabovskis A, Aivars J-I. Bilateral difference of superficial and deep femoral artery haemodynamic and anatomical parameters.Artery Res. 2013;7: 201-210. doi:10.1016/j.artres.2013.09.001

40. Mayrovitz HN, Larsen PB. Pulsatile blood flow asymmetry in paired human legs.Clin Physiol. 1996;16: 495-505. doi:10.1111/j.1475-097X.1996.tb01015.x

41. Climie RE, Gallo A, Picone DS, Di Lascio N, van Sloten TT, Guala A, et al., Measuring the Interaction Between the Macro- and Micro-Vasculature.Front. Cardiovasc. Med. 2019; 6:169.doi: 10.3389/fcvm.2019.00169

42. Sciomer S, Moscucci F, Dessalvi CC, Deidda M, Mercuro G. Gender differences in cardiology: is it time for new guidelines? J Cardiovasc Med (Hagerstown). 2018 Dec;19(12):685-688. doi: $10.2459 / \mathrm{JCM} .0000000000000719$.

43. Hunter SK. The Relevance of Sex Differences in Performance Fatigability. Med Sci Sports Exerc. 2016 Nov;48(11):2247-2256. doi: 10.1249/MSS.0000000000000928.

44. Boit, M.D. , Sibson, R. , Meakin, J.R. , Aspden, R.M. , Thies, F. , Mangoni, A.A., et al., Sex differences in the response to resistance exercise training in older people. Physiol Rep, 4 ( 12), 2016, e12834, doi: $10.14814 /$ phy2.12834

45. Ansdell, P, Thomas, K, Hicks, KM, Hunter, SK, Howatson, G, Goodall, S. Physiological sex differences affect the integrative response to exercise: acute and chronic implications. Experimental Physiology.

2020; 105, 2007- 2021. https://doi.org/10.1113/EP088548 
Figure Legends:

Figure 1: Representative signal recordings obtained in the present experimental conditions. Dorsal foot perfusion records in one participant obtained from LDF (top) PPG (middle) and TiVi (bottom) systems illustrate the different interactions of the light with the tissue. The final fifteen seconds of Phase 1 and the initial fifteen seconds of Phase 2 are shown (see text).

Figure 2: Normal distribution of baseline data for the perfusion related variables obtained from (a) PPG (b) LDF and (c) TiVi, in Groups I and II (see text).

Figure 3.

Graphic illustration of the most representative perfusion related variables obtained in male and female participants ( $n=12)$, independent of age, in the studied experimental conditions. Indicated values represent the respective mean $(\underline{t} \mathrm{sd})$ of each participant's limb pair by sex. No significant differences were found (see text). 
LDF

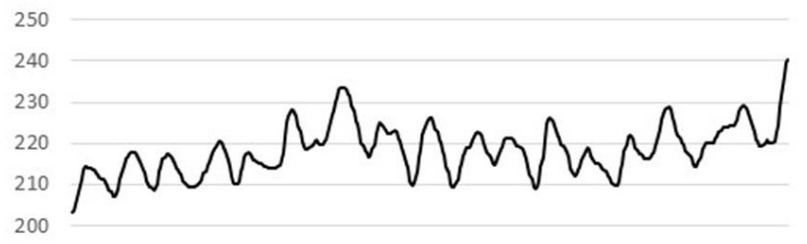

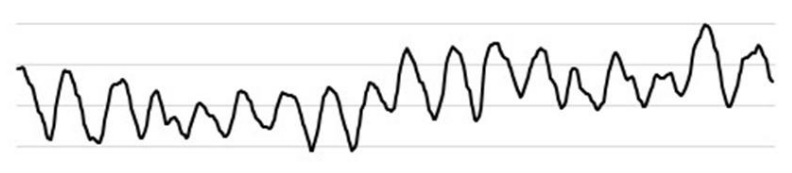
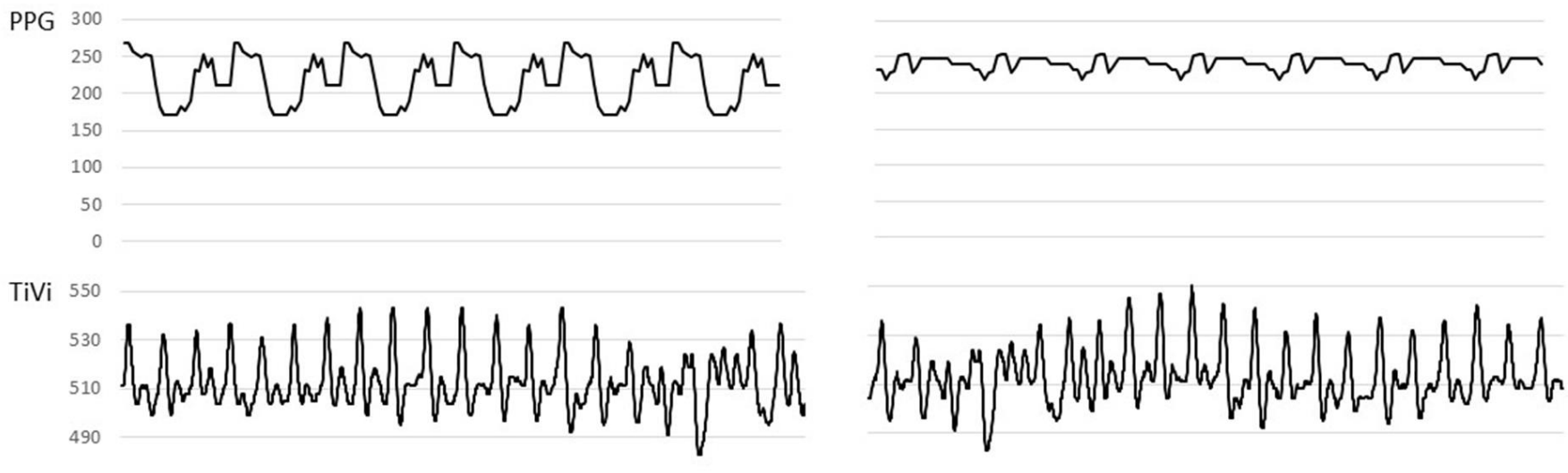

470

450

Phase 1

Phase 2 

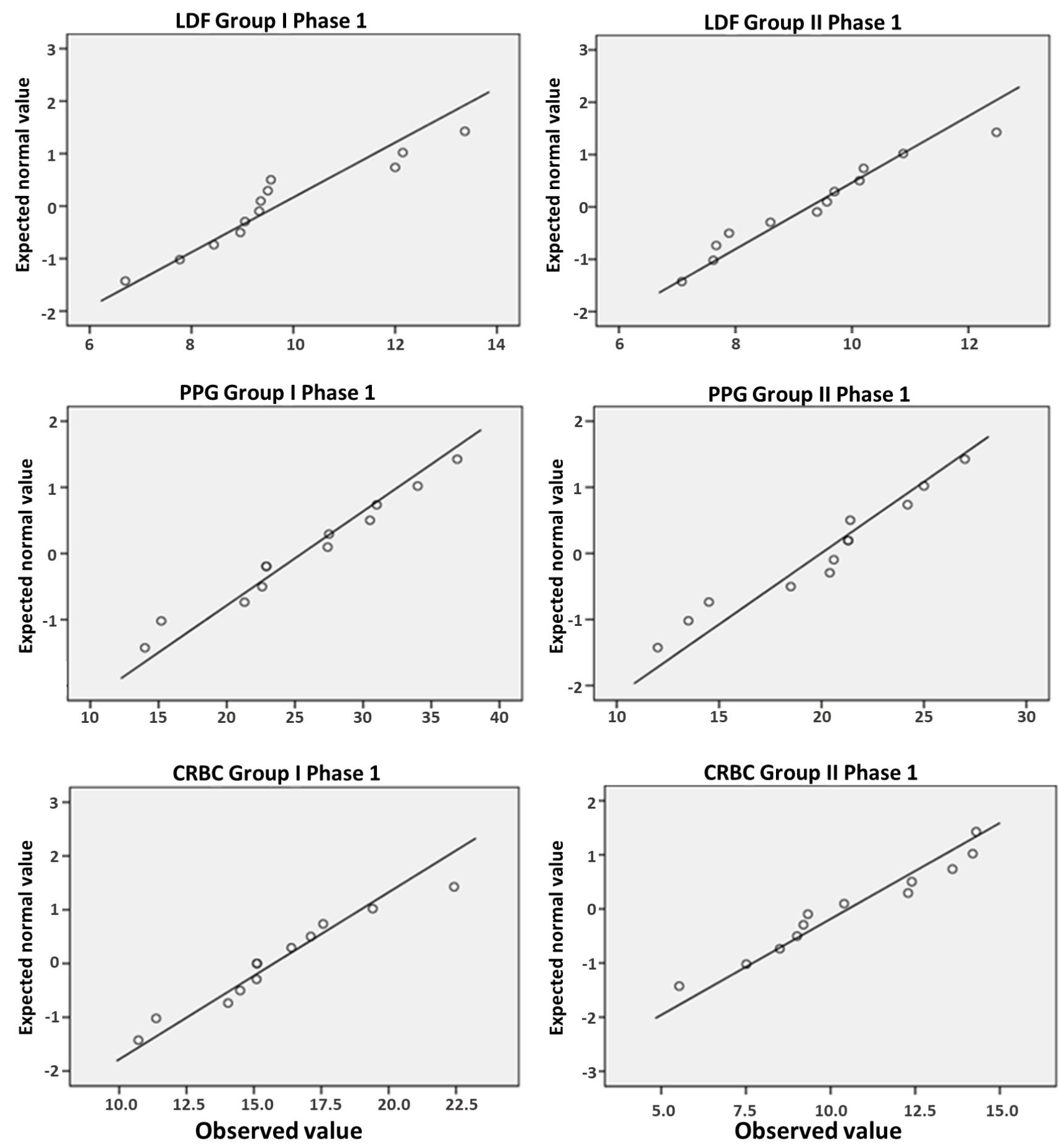


\section{perfusion (BPUs)}

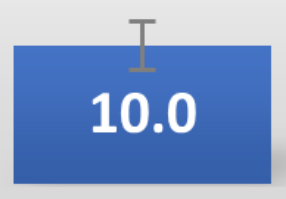

Phase 1

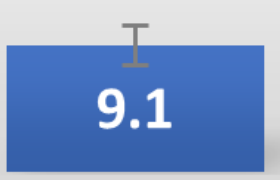

Phase 2

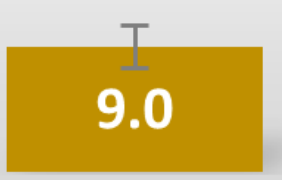

Phase 1

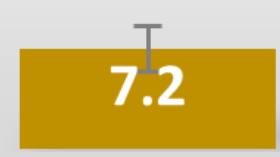

Phase 2

Male

Female

\section{velocity $(\mathrm{cm} / \mathrm{s})$}

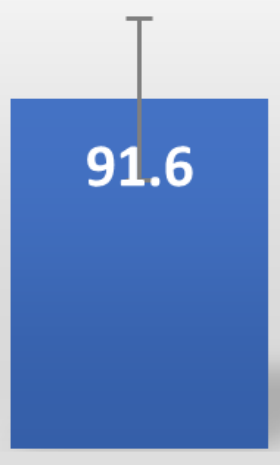

Phase 1

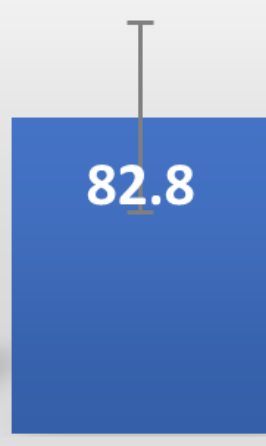

Phase 2

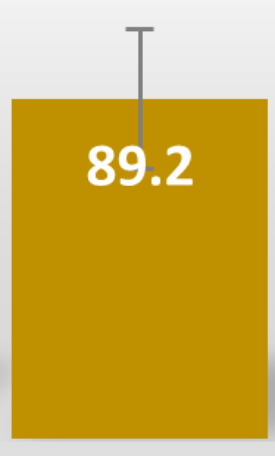

Phase 1

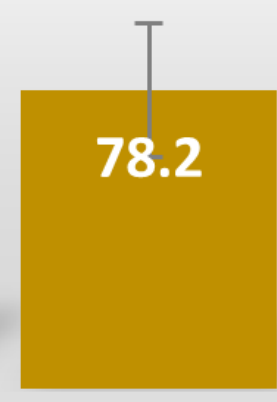

Phase 2

Male

Female

\section{TIVI (AU)}

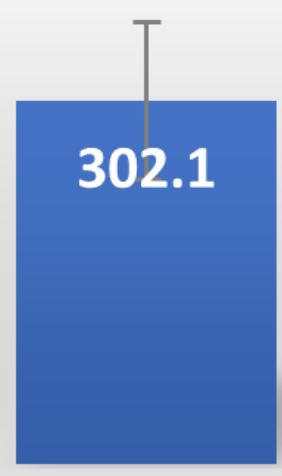

Phase 1

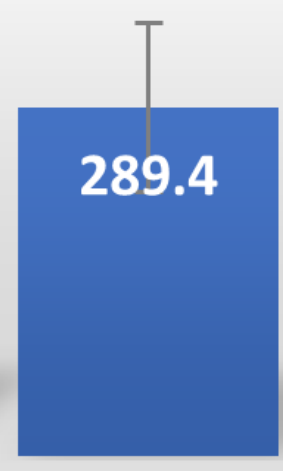

Phase 2

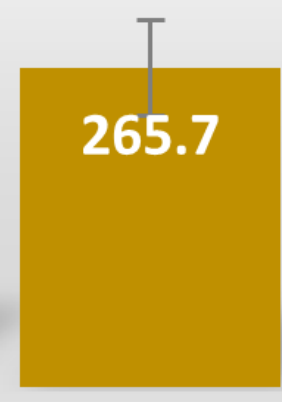

Phase 1

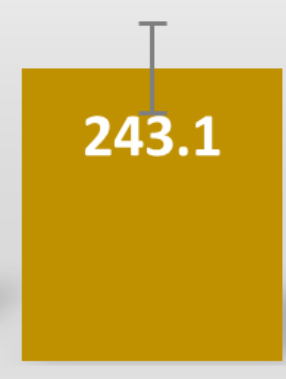

Phase 2 
Table 1. Mean lower limb perfusion changes expressed in arbitrary units (BPU, AU) obtained by LDF, PPG and TiVi in all participants' left and right lower limbs during the experimental protocol. Data are expressed as means \pm standard deviations ( $s d)$, followed by $p$ values where applicable; $(n=12)$. Intraindividual statistical comparison ( $t$ test) between right and left limbs is shown in each phase. In each group, a statistical comparison between phases is also shown (Wilcoxon sign rank test; ${ }^{*} \mathrm{p}<0.05 ; \mathrm{ns}-$ non significant).

\begin{tabular}{|c|c|c|c|c|c|c|c|c|c|}
\hline & & \multicolumn{2}{|c|}{ Phase 1} & \multicolumn{2}{|c|}{ Phase 2} & \multicolumn{2}{|c|}{ Phase 1} & \multicolumn{2}{|c|}{ Phase 2} \\
\hline & & Right & Left & Right & Left & Right & Left & Right & Left \\
\hline \multirow{3}{*}{$\begin{array}{c}\text { LDF } \\
\text { Perfusion } \\
\text { (BPU) }\end{array}$} & mean $\pm s d$ & $10.4 \pm 1.9$ & $9.0 \pm 1.8$ & $8.9 \pm 2.2$ & $7.7 \pm 2.2$ & $9.2 \pm 1.4$ & $9.4 \pm 1.9$ & $8.1 \pm 1.7$ & $8.1 \pm 1.3$ \\
\hline & $p$-value & \multicolumn{2}{|c|}{ ns } & \multicolumn{2}{|c|}{ ns } & \multicolumn{2}{|c|}{ ns } & \multicolumn{2}{|c|}{ ns } \\
\hline & $\begin{array}{l}\text { ₹ phases } \\
\text { (p-value) }\end{array}$ & \multicolumn{4}{|c|}{$0.033 *$} & \multicolumn{4}{|c|}{$0.028 *$} \\
\hline \multirow{4}{*}{$\begin{array}{l}\text { CRBC } \\
(A U)\end{array}$} & mean $\pm s d$ & $16.6 \pm 3.8$ & $14.8 \pm 2.5$ & $17.6 \pm 9.3$ & $18.5 \pm 9.1$ & $11.4 \pm 3.0$ & $10.0 \pm 2.8$ & $10.7 \pm 3.5$ & $9.5 \pm 2.4$ \\
\hline & p-value & \multicolumn{2}{|c|}{ ns } & \multicolumn{2}{|c|}{ ns } & \multicolumn{2}{|c|}{ ns } & \multicolumn{2}{|c|}{ ns } \\
\hline & $\begin{array}{l}\text { ₹ phases } \\
\text { (p-value) }\end{array}$ & \multicolumn{4}{|c|}{0.272} & \multicolumn{4}{|c|}{0.583} \\
\hline & mean $\pm s d$ & $72.5 \pm 2.6$ & $90.2 \pm 1.4$ & $76.0 \pm 2.0$ & $82.8 \pm 6.3$ & $92.0 \pm 7.7$ & $106.8 \pm .4$ & $84.0 \pm 13.2$ & $79.3 \pm 3.4$ \\
\hline \multirow{2}{*}{$\begin{array}{l}\text { Velocity } \\
(\mathrm{cm} / \mathrm{sec})\end{array}$} & $p$-value & \multicolumn{2}{|c|}{ ns } & \multicolumn{2}{|c|}{ ns } & \multicolumn{2}{|c|}{ ns } & \multicolumn{2}{|c|}{ ns } \\
\hline & $\begin{array}{l}\neq \text { phases } \\
\text { (p-value) }\end{array}$ & \multicolumn{4}{|c|}{ ns } & \multicolumn{4}{|c|}{$0.003^{*}$} \\
\hline \multirow{3}{*}{$\begin{array}{c}\text { PPG } \\
B V P \\
(A U)\end{array}$} & mean $\pm s d$ & $27.7 \pm 8.2$ & $23.4 \pm 5.6$ & $23.9 \pm 8.0$ & $23.2 \pm 6.7$ & $21.3 \pm 4.5$ & $18.7 \pm 4.8$ & $19.0 \pm 6.2$ & $18.2 \pm 5.0$ \\
\hline & p-value & \multicolumn{2}{|c|}{0.315} & \multicolumn{2}{|c|}{0.879} & \multicolumn{2}{|c|}{0.352} & \multicolumn{2}{|c|}{0.849} \\
\hline & $\begin{array}{l}\text { f phases } \\
\text { (p-value) }\end{array}$ & \multicolumn{4}{|c|}{ ns } & \multicolumn{4}{|c|}{ ns } \\
\hline \multirow{2}{*}{$\begin{array}{l}\text { Pulse } \\
\text { Rate } \\
\text { (bpm) }\end{array}$} & mean $\pm s d$ & & & & & 73.8 & 10.0 & 76.2 & \\
\hline & $\begin{array}{l}\neq \text { phases } \\
\text { (p-value) }\end{array}$ & & & & & & & & \\
\hline PSp & mean \pm sd & $\begin{array}{c}322.5 \pm \\
66.0\end{array}$ & $\begin{array}{c}289.3 \pm \\
60.8\end{array}$ & $\begin{array}{c}295.1 \pm \\
63.8\end{array}$ & $\begin{array}{c}297.7 \pm \\
71.1\end{array}$ & $\begin{array}{c}270.2 \pm \\
35.9\end{array}$ & $\begin{array}{c}253.6 \pm \\
44.6\end{array}$ & $244.9 \pm 36.2$ & $227.2 \pm 22.0$ \\
\hline CRBC & p-value & & & & & & & & \\
\hline & $\begin{array}{l}\neq \text { phases } \\
\text { (p-value) }\end{array}$ & & & & & & & & \\
\hline SysP & mean $\pm s d$ & 118. & 11.7 & 121 & 13.7 & 113. & 19.9 & 114.0 & 17.5 \\
\hline$(\mathrm{mmHg})$ & $\begin{array}{l}\neq \text { phases } \\
\text { (p-value) }\end{array}$ & & & & & & & & \\
\hline DiastP & mean \pm sd & 68. & & & & & 5.1 & 74.2 & \\
\hline$(\mathrm{mmHg})$ & $\begin{array}{l}\neq \text { phases } \\
\text { (p-value) }\end{array}$ & & & & & & & & \\
\hline
\end{tabular}


Table 2. Comparing most representative perfusion related variables obtained as a function of age in the present experimental conditions. Each participant's $(n=12)$ limb pair was transformed in one single normalized value corresponding to their mean $( \pm s d)$. Comparison between groups and study phases is followed by $p$ values where applicable (ns - non significant; $\left.{ }^{*} p<0.05\right)$.

\begin{tabular}{|c|c|c|c|c|c|}
\hline & & \multicolumn{2}{|c|}{ Phase 1} & \multicolumn{2}{|c|}{ Phase 2} \\
\hline & & Group I & Group II & Group I & Group II \\
\hline \multirow{3}{*}{$\begin{array}{c}\text { LDF } \\
\text { Perfusion } \\
\text { (BPU) }\end{array}$} & mean $(\underline{+} \mathrm{sd})$ & $9.7(1.4)$ & $9.3(1.5)$ & $8.3(2.0)$ & $8.1(1.0)$ \\
\hline & $\neq$ group (p-value) & \multicolumn{2}{|c|}{0.589} & \multicolumn{2}{|c|}{0.937} \\
\hline & $\neq$ phase(p-value) & _- & _- & $0.046^{*}$ & $0.046^{*}$ \\
\hline \multirow{3}{*}{$\begin{array}{l}\text { Velocity } \\
\text { (cm/ sec) }\end{array}$} & mean (sd) & $81.4(18.1)$ & $99.4(8.6)$ & $79.4(21.7)$ & $81.7(15.1)$ \\
\hline & $\neq$ group ( $p$-value) & \multicolumn{2}{|c|}{0.093} & \multicolumn{2}{|c|}{0.583} \\
\hline & $\neq$ phase(p-value) & - & _- & 0.345 & $0.028^{*}$ \\
\hline PSp & mean (sd) & $305.9(56.1)$ & $261.9(36.3)$ & $296.4(64.2)$ & $236.1(19.2)$ \\
\hline CRBC & $\neq$ group ( $p$-value) & \multicolumn{2}{|c|}{0.818} & \multicolumn{2}{|c|}{0.589} \\
\hline$(A U)$ & $\neq$ phase(p-value) & - & _ & 0.075 & $0.046^{*}$ \\
\hline
\end{tabular}

